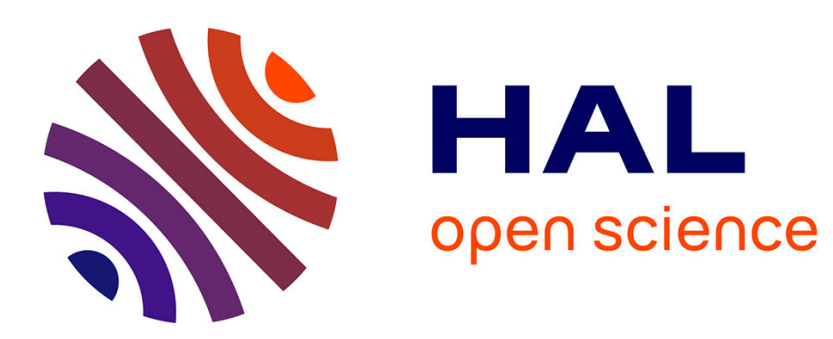

\title{
GAIN ENHANCEMENT OF DIELECTRIC RESONATOR LOADED WAVE-GUIDE ANTENNAS WITH DIELECTRIC OVERLAYS
}

Mohammad Hakkak, Hossein Ameri Mahabadi

\section{- To cite this version:}

Mohammad Hakkak, Hossein Ameri Mahabadi. GAIN ENHANCEMENT OF DIELECTRIC RESONATOR LOADED WAVE-GUIDE ANTENNAS WITH DIELECTRIC OVERLAYS. Electronics Letters, 1992, 28 (6), pp.541-542. hal-00714698

\section{HAL Id: hal-00714698 \\ https://hal.science/hal-00714698}

Submitted on 5 Aug 2012

HAL is a multi-disciplinary open access archive for the deposit and dissemination of scientific research documents, whether they are published or not. The documents may come from teaching and research institutions in France or abroad, or from public or private research centers.
L'archive ouverte pluridisciplinaire HAL, est destinée au dépôt et à la diffusion de documents scientifiques de niveau recherche, publiés ou non, émanant des établissements d'enseignement et de recherche français ou étrangers, des laboratoires publics ou privés. 
model correctly describes the electromagnetic nature of the media throughout the considered frequency bandwidth.

22nd January 1992

Ph Leveque A Reineix and B Jecko (IRCOM-Equipe 'Electromagnétisme', Faculté des Sciences-URA au CNRS no. 356, 123 avenue Albert Thomas, 87060 Limoges Cedex, France)

References

1 REINEIX, A., and JECKO, B.: 'Analysis of microstrip patch antennas using finite difference time domain method', IEEE Trans., 1989, AP-37, (11), pp. 1361-1369

2 LUEBBERS, R., HUNSBERGER, F. P., KUNZ, K. S., STANDLER, R. B., and SCHNEIDER, M.: 'A frequency-dependent finite-difference timedomain formulation for dispersive materials', IEEE Trans., 1990, EC-32, (3), pp. 222-227

3 BUI, M. D., STUCKLy, s. S., and Costache, G. 1.: 'Propagation of transients in dispersive dielectric media', IEEE Trans., 1991 MTT-39, (7), pp. 1165-1172

4 VAUDON, P., REINEIX, A., and JECKO, B.: 'Asymptotic method in prediction of E-plane radiation pattern of rectangular microstrip patch antennas of finite ground', Electron. Lett., 1990, 26, (13), pp. 956-957

\section{GAIN ENHANCEMENT OF DIELECTRIC RESONATOR LOADED WAVEGUIDE ANTENNAS WITH DIELECTRIC OVERLAYS}

M. Hakkak and H. Ameri

Indexing terms: Antennas, Dielectric and dielectric devices, Resonators, Antenna radiation patterns

The gain characteristics of a dielectric resonator loaded coaxial probe fed circular waveguide antenna (DRLWA) with overlaying parasitic discs have been investigated experimentally. Results indicate that, when properly spaced, the overlays can enhance the gain by more than $6 \mathrm{~dB}$.

Introduction: Dielectric resonator antennas (DRAs) have recently been proposed [1] as simple efficient nonmetallic radiators, especially useful for microwave and millimetre-wave bands. Recently, it was also shown [2] that when the dielectric resonator is embedded inside a circular waveguide the radiation pattern of the waveguide aperture may be further improved by optimising the relative dielectric and waveguide dimensions. In particular, the waveguide dimension adjustment helps in tuning the resonance frequency to a desired one. In this Letter, experimental results on the effect of overlays on the gain of the latter radiator are reported. It may be mentioned that in a study in this area, Lee and Lee [4] and Afzalzadeh [5] noticed a significant improvement in the gain of bare microstrip antennas with parasitic overlays, a matter that prompted the present investigation.

Experimental results: A typical DRLWA is shown in Fig. 1. In the present study the dielectric disc was chosen to have $\varepsilon_{r}=8$ and dimensions for resonance frequency of $11 \mathrm{GHz}$. The disc

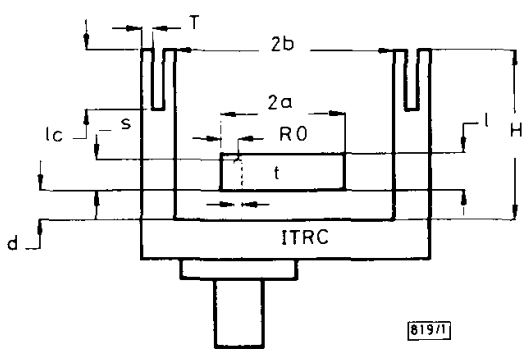

Fig. I Geometry is excited by a coaxial probe so that the input impedance is nearest to $50 \Omega$. When the disc is encapsulated in a waveguide of internal diameter $22.3 \mathrm{~mm}$ the resonance frequency shifted to $11.35 \mathrm{GHz}$. As mentioned in Reference 3, the input impedance for the probe can be predicted by solving for the excited modes after determination of the proper Green function of this particular arrangement. The $\lambda / 4$ choke is introduced to reduce both sidelobe and crosspolarisation levels of the waveguide radiation pattern. Fig. 2 shows the E-plane pattern with a beamwidth of approximately $52^{\circ}$.

When parasitic elements are placed above the waveguide aperture, an appreciable increase in the forward gain is observed. To study the effect the various models shown in Fig. 3 were examined. In all these models, discs of $\varepsilon_{r}=4$ and diameter $6.6 \mathrm{~cm}$ corresponding to $3.9 \lambda$ were found to be the smallest suitable to yield the best compromise for gain increase and sidelobe level decrease. Therefore in this study this

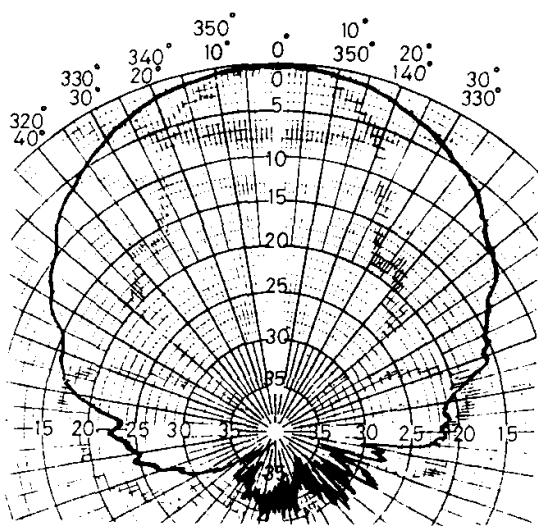

$819 / 2$

Fig. 2 E-plane radiation pattern of DRLWA

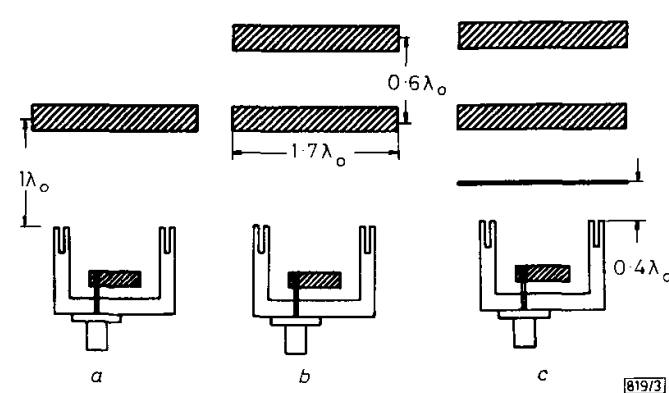

Fig. $3 D R L W A$ with overlays

a Single overlay $\varepsilon_{r}=4$

Double overlay $\theta_{r}=4$

c Double overlay $\varepsilon_{r}=4$ with additional $\varepsilon_{r}=2.33$ parasitic

parameter was fixed and attention was focused on the effect of distance above the aperture. Moreover, the thickness of the parasitic discs was chosen to be $\lambda_{g} / 2$ to avoid strong multiple reflection effect within the dielectric and thus allow maximum transmission through it.

The best pattern obtained from model $a$ in Fig 3 in which the lowest sidelobe occurred when the disc was at a distance $\lambda_{0}$ is shown in Fig. 4. The first sidelobe has a level of about $17 \mathrm{~dB}$ and also a large cluster sidelobe of the same level near the horizon. When another parasitic element was added (model $b$ of Fig. 3) the first sidelobe was smoothed and a slight increase in gain observed. However, the horizontal lobe still persisted. To reduce the level of this sidelobe, a thin PTFE disc of the same diameter was introduced in the vicinity of the aperture (model c). In this way we could reduce all sidelobe levels below about $22 \mathrm{~dB}$. The gain of this model turns out to be about $12 \mathrm{~dB}$. 
The above characteristics were observed to remain almost constant for at least $10 \%$ bandwidth. The return loss was better than $14 \mathrm{~dB}(V S W R<1.6)$ for a wider bandwidth, however.

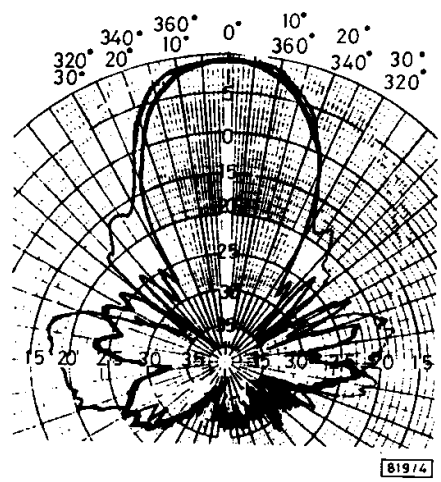

Fig. 4 Radiation pattern of DRLW A with dielectric overlays

Conclusion: An experimental study for gain enhancement of dielectric resonator loaded waveguide antennas using parasitic dielectric directors has been presented. Results indicate that, with two directors a gain improvement of about $7 \mathrm{~dB}$ or more may be obtained over a bandwidth of at least $10 \%$. The whole unit may be packaged inside lower dielectric capsule with elements placed properly using foam spacers.

I4th January 1992 M. Hakkak and H. Ameri (Iran Telecom Research Center, PO Box 14155-3961, Tehran 14394, Iran)

\section{References}

1 LONG, S. A., MCALlister, M. W., and SHEN, L. C.: 'The resonant cylindrical dielectric cavity antenna', IEEE Trans., 1983, AP-31, pp. 406-412

2 KISHK, A. A., AUDA, A. A., and AHN, B. C.: 'Radiation characteristics of cylindrical dielectric resonator antennas with new applications' IEEE Antennas and Propagation Society Newsletter, February 1989 , pp. $7-16$

3 Safavi-Nain, s., and ameru, $H .:$ 'Modal analysis of dielectric loaded coaxial probe fed circular waveguide radiator'. IEEE/AP-S Symp. London, Ontario, Canada, June 1991

4 LEE, R. Q., and LEE, K. F.: 'Gain enhancement of microstrip antennas with overlaying parasitic directors', Electron. Lett., 1988, 24, (11) pp. 656-658

5 AFZALZADEH, R.: 'Studies on the effects of overlays on microstrip patch antenna and reflection filter'. PhD Thesis, University of Poona, India, December 1990

\section{PREFORMING AN ELECTROMAGNETIC} PULSE IN LOSSY MEDIUM

J. R. Wait and K. A. Nabuls

\section{Indexing terms: Electromagnetic waves, Wave propagation}

Plane wave propagation of a transient signal in a lossy homogeneous medium is considered. It is shown that it is possible to prescribe a source signal waveform which will produce a Gaussian signal waveform after propagating a specified distance.

As is well known, a transient signal propagating in lossy media suffers both attenuation and waveform distortion [1] We pose the question: can the source signal waveform be tailored to produce an impulse after propagating a given distance in the medium? A solution for an ideal case is presented here. In some sense, the problem is analogous to the design of matched filters in radar signal processing [2]

We consider an unbounded homogeneous region of permittivity $\varepsilon$, conductivity $\sigma$ and magnetic permeability $\mu$. We deal with one dimensional plane wave propagation in the positive $z$ direction. Thus the transverse derivatives $\partial / \partial x$ and $\partial / \partial y$ are zero. The electric field is taken to have only an $x$ component $e_{x}(z, t)$ and, henceforth, the subscript is dropped.

Let us now say, at $z=z_{0}$, the desired signal is the Gaussian pulse

$$
\left.e\left(z_{0}, t\right)=e_{0} \exp -\beta^{2} t^{2}\right)
$$

being defined for all time, $-\infty<t<\infty$, where $e_{0}$ and $\beta$ are constants. The width of the pulse is $1 / \beta$ seconds. Noting that $z_{0}>0$, the objective is to determine the source signal $e_{s}(t)=$ $e(0, t) / e_{0}$ at $z=0$ which will produce the desired Gaussian pulse at $z=z_{0}$

To proceed we take Fourier transforms as follows:

$$
\begin{aligned}
& E\left(z_{0}, j \omega\right)=\int_{-\infty}^{+\infty} e\left(z_{0}, t\right) e^{-j \omega t} d t \\
& E(0, j \omega)=\int_{-\infty}^{+\infty} e(0, t) e^{-j \omega t} d t
\end{aligned}
$$

Now clearly

$$
E\left(z_{0}, j \omega\right)=E(0, j \omega) \exp \left[-\gamma(j \omega) z_{0}\right]
$$

where

$$
\chi(j \omega)=[j \mu \omega(\sigma+j e \omega)]^{1 / 2}
$$

is the propagation factor for plane wave transmission at an angular frequency $\omega$.

The desired solution is the inverse Fourier transform

$$
\begin{aligned}
e(0, t)= & \frac{e_{0}}{2 \pi} \int_{-\infty}^{+\infty} E\left(z_{0}, j \omega\right) \\
& \times \exp \left[\gamma(j \omega) z_{0}+j \omega t\right] d \omega
\end{aligned}
$$

where, on employing eqns. 1 and 2 , we deduce that

$$
E\left(z_{0}, j \omega\right)=\left[e_{0} \sqrt{ }(\pi) / \beta\right] \exp \left(-\omega^{2} / 4 \beta^{2}\right)
$$

Eqn. 6 may then be written in the form

$$
\begin{aligned}
e_{s}(t)= & \frac{1}{\beta \sqrt{ }(\pi)} \int_{0}^{\infty} \exp \left[R(\omega) z_{0}-\left(\omega^{2} / 4 \beta^{2}\right)\right] \\
& \times \cos \left[I(\omega) z_{0}+\omega t\right] d \omega
\end{aligned}
$$

where $\boldsymbol{R}(\omega)+j I(\omega)=\gamma(j \omega)$. Here we have also used $\boldsymbol{R}(-\omega)=\boldsymbol{R}(\omega)$ and $\boldsymbol{I}(-\omega)=-I(\omega)$

In the relatively trivial case, where $\sigma=0$ and both $\varepsilon$ and $\mu$ are independent of frequency, $\gamma(j \omega)=j \omega / v$ where $v=(\varepsilon \mu)^{-1 / 2}$. On using eqn. 6, we obtain

$$
e(0, t)=e_{0} \exp \left[-\beta^{2}\left(t+\frac{z_{0}}{v}\right)^{2}\right]
$$

which is also a Gaussian pulse with a maximum at $t=-z_{0} / v$ seconds.

When the conductivity is not zero, the integral given by eqn. 8 needs to be evaluated numerically. Results for $e_{s}(t)=$ $e(0, t) / e_{0}$ are shown in Fig. 1 as a function of $\beta t$ where $\beta=10^{7} \mathrm{~s}^{-1}, z_{0}=1 \mathrm{~m}, \varepsilon / \varepsilon_{0}=\varepsilon_{r}=10, \mu_{r}=\mu / \mu_{0}=1$, and $\sigma=0,1,5,10$, and $50 \mathrm{mS} / \mathrm{m}$. The zero conductivity case replicates the desired signal Gaussian pulse $e\left(z_{0}, t\right)$ in accordance

ELECTRONICS LETTERS 12th March 1992 Vol. 28 No.6 